ISSN:2641-1725

\title{
Shockwave Therapy in Atherosclerosis
}

\author{
Michael AB Naafs* \\ Endocrinologist, Health Consultant at Naafs, International Health Consultancy, Rhodoslaan 20,7577KN, Oldenzaal, Netherlands
}

Received: 䟧 August 16, 2018; Published: 些 August 21, 2018

*Corresponding author: Michael AB Naafs, Endocrinologist, Health Consultant at Naafs, International Health Consultancy, Rhodoslaan 20,7577KN, Oldenzaal, Netherlands

\begin{abstract}
In this mini review recent developments in extracorporeal shockwave therapy (ESWT) within the field of atherosclerosis are discussed. Increased ecpression of growth factors such as endothelial nitric oxide (eNOS) and vascular endothelial growth factors (VGEFs) are induced by ESWT. IKL, integrin linked kinase, plays a key factor in this process of angiogenesis, believed to be responsible for the beneficial effects of ESWT. Both cardiac shock wave therapy (CWST) in chronic refractory angina pectoris patients as well as ESWT in patients with peripheral arterial disease (PAD) show a uniformly, consistent, beneficial effect in all studies available until now. International standardization of dose-response curves, doses applied, number of sessions and duration of treatment should be established at first, before starting large RCTs. With that knowledge the future of shockwave therapy in CAD and PAD seems bright.
\end{abstract}

\section{Introduction}

The medical use of high energy extracorporeal shockwaves started in the 1980's, when they were targeted to fragment urinary stones (lithotripsy, ESWL) and gallstones [1,2]. In addition ESWT has been used in orthopedics for musculoskeletal disorders, wound and non-union healing of fractures and the stimulation of bone growth [3-8]. The exact mechanism of action of ESWT on soft tissue is not fully understood, although the principle of mechano transduction is thought to be responsible for the biological changes that take place, Mechano transduction transfers mechanical stimuli into chemical signals at the cellular level, which activates gene expression of growth factors and cytokines as well as cell proliferation and differentiation [9]. Increased expression of growth factors such as endothelial nitric oxide synthase (eNOS) and vascular endothelial growth factors (VGEFs) in soft tissue has been observed in animal studies [10-13]. Due to these findings further applications of ESWT are explored such as in atherosclerosis. One such application included the use of ESWT in ischaemic heart disease and in claudication in peripheral arterial disease. In this mini-review the recent developments in the application of ESWT in the field of atherosclerosis are discussed.

\section{Cardiac Shockwave Therapy (CSWT)}

The anti -anginal efficacy of cardiac shock wave therapy was investigated in a Cochrane analysis by Burneikaite et al. [14]. In total 39 studies evaluating the efficacy of CSWT in patients with stable angina were identified including single arm, non- and randomized trials. Totally, 1189 patients were included in 39 reviewed studies with 1006 patients treated with CSWT. The largest patient sample consisted of 111 patients. All selected studies demonstrated significant improvement in subjective measures of angina pectoris and or quality of life. In the majority of studies left ventricular function and myocardial perfusion improved. In 12 controlled studies with 483 patients included (183 controls) angina class, Seattle Angina Questionnaire (SAQ) score and nitrate consumption were significantly improved after the treatment. In 593 participants across 22 studies the exercise capacity was significantly improved after CSWT, as compared with the baseline values (in meta-analysis standardized mean difference SMD=;-0,74;95 CI:-0.97 to-0,5; $\mathrm{p}<0,001)$.The authors conclude that CSWT in stable coronary artery disease (CAD) demonstrated consistent improvements of clinical variables. Meta-analysis showed a moderate improvement of exercise capacity. Overall, CSWT is a promising non-invasive option for patients with end-stage CAD but evidence is limited to small sample, single center studies [14].

Yang showed in a rat model of acute myocardial infarction (AMI) that CSWT improves myocardial perfusion and ameliorates cardiac remodeling [15]. They demonstrated the arteriogenesis of coronary microangium and protein expression change in ischaemic myocardium after CSWT. Four weeks after CSWT, the fractioning of rats was improved greatly and the cardiomyocyte apoptosis index was significantly lower than in the AMI group $(p<0,05)$. Besides, the fibrotic area was markedly decreased in the CSWT group. In the 
infarction borderzone, the thickness of smooth muscle layer was expanded apparently after CSWT. Label-free quantitative proteomic analysis and bioinformatics analysis revealed that the differentially exposed proteins were largely enriched in the focal adhesion signalling pathway. Integrin linked kinase (ILK) may be a key factor contributing to arteriogenesis of coronary micrangium during CSWT [15]. Vainer studied 33 patients with chronic refractory angina pectoris and reverible ischaemia on myocardial scintigraphy. CSWT was applied to the ischaemic zones (3-7 spots,100 impulses/ spot,0,09 $\mathrm{mJ} /$ square $\mathrm{mm}$ ) in an echocardiography -guided and ECG- triggered fashion [16]. The protocol included a total of 9 treatment sessions (3 sessions within 1 week at baseline, and after 1 and 2 months. Clinical measurement was performed using exercise testing,angina score (CCS class), nitrate use,myocardial scintigraphy, and cardiac magnetic resonance (CMR) 1 and 4 months after the last treatment session. One and 4 months after CSWT, sublingual nitrate use decreased from 10 /week to 2 /week $(p<0,01)$ and the angina symptoms diminished from CCS class 3 to CCS class $2(p<0,01)$. This clinical improvement was accompanied by an improved myocardial uptake on stress myocardial scintigraphy $(54,2$ plus/minus $7,7 \%$, to $56,4+9,4 \% ; p=0,016)$ and by increased exercise tolerance at 4-month follow-up (from 7,4 plus minus 2,8 to 8,8 plus minus $3,6 \mathrm{~min}: \mathrm{P}=0,015)$. No clinically relevant side effects were observed [16].

Shkollink designed a model for a randomized, triple blind, sham procedure, controlled study to reach a better quality evidence of CSWT in patients with refractory angina pectoris despite optimal medical treatment, responsible for $20 \%$ of cardiac deaths in Europe [17]. The new study protocol aims to extend the widespread use of CSWT that is not based on the results of imaging tests or coronary angiography,which are unavailable sometimes. Until now CSWT is applied to the ischaemic segments of the Left Ventricle (LV). This new study design foresees in application of CSWT to all segments of LV which may provide beneficial therapeutic effects by not only reducing ischaemia but also attenuating inflammation and suppressing oxidative stress and fibrosis in non-ischaemic segments, as well potentially preventing LV remodeling [17]. More multicenter trials of CSWT are underway [18].

\section{ESWT and Peripheral Arterial Disease (PAD)}

PAD has a prevalence of $4,6 \%$ and of $20 \%$ in people older than 60 years with intermittent claudication (IC) as one of the earliest and most common symptoms. PAD has detrimental effects on patients' walking ability in terms of maximum walking distance (MWD) and pain-free walking distance (PFWD). Research has suggested that ESWT may induce angiogenesis in treated tissue. PAD sufferers experience a cramping pain in the affected muscles, most commonly the calves, which occurs after a short walking distance, until the patient stops, where the pain then gradually subsides. PAD can be limiting and occasionally debilitating and has been shown to have considerable effects on patients 'quality of life [19]. To date 4 studies have investigated ESWT in claudication. In a randomized trial of 22 patients (12 patients treated,10 controls) Ciccone and
Notarnicola targeted ESWT at a stenotic plaque lesion within lower limb arteries and demonstrated significant reduction in degree of arterial stenosis,significant improvement in PFWD and reduction in pain severity. These findings were also coupled with a significant reduction in Fontaine staging, In this study ESWT was applied in 4 sessions at 1 weekly interval (2000 impulses, $0,03 \mathrm{~mJ} /$ square $\mathrm{mm}$ )- [20]. Tara and Miyamota performed a small prospective non-randomized study $(\mathrm{n}=10)$ which aimed to prove safety and its primary end-point was observation of adverse events [21]. The study found no adverse events secondary to shockwave therapy. The secondary end -point was a measure of tissue perfusion using scintigraphy, laser Doppler and measurement of transcutaneous oxygen tension (TcP02). The use of scintigraphy improved tissue perfusion in the dorsum of the foot. However, perfusion was unchanged in the lower leg region. Moreover, TcPO2 increased significantly in both the calf region and dorsum of the foot. Skin perfusion pressure using laser Doppler showed increases in both regions, but neither was significant [21]. Belcaro and Cesarone studied 32 patients undergoing ESWT for critical limb ischaemia with both rest pain and localized distal necrosis [22]. Outcome included PFWD, visual analogue scale (VAS) for pain, laser Doppler skin perfusion, partial pressure of oxygen and partial pressure of carbon dioxide. All outcomes demonstrated significantly improved measurements [22]. Serizawa et al performed two prospective nonrandomized trials with a total of 6 patients and then 12 patients with Fontaine stage 2 PAD who were treated with ESWT. Patients demonstrated significantly maximum walking distance (MWD), shorter recovery time and improved walking questionnaire scores [23].

Studies in PAD not caused by atherosclerosis as e.g.in patients with thromboangiitis obliterans (Buerger's disease are not known yet. As dose response curves and standardization are nearly lacking ESWT regimen differ enormously in doses apllied, number of sessions and duration of treatment. New trials such as the Shockwave 1 trial are enrolling patients now to meet some of the shortcomings in present available data [24].

\section{Conclusion}

It is obvious that extracorporeal shockwave therapy will be a promising treatment modality in end-stage coronary artery diseases (CAD) and in peripheral arterial disease (PAD) Patients with chronic refractory angina pectoris, not amenable to bypass surgery or catheter intervention therapy seem to benefit from cardiac shock wave therapy (CSWT). The same picture is seen in PAD patients, especially those with crural artery disease of the lower limbs. The results of bypass surgery or catheter interventional therapy for infrapoplitial lesions are still unsatisfactory and recurrence rates are high in these patients.

Until now study samples are small and large RCTs should be performed in the future. Nevertheless, all these available studies show a consistent uniformly beneficial picture, even in a Cochrane analysis of CSWT. It is clear that the first goal should be establishing 
international standardization of dose-response- curves, doses applied, number of sessions and duration of treatment. With that knowledge extracorporeal shock wave therapy will have a bright future in CAD and PAD.

\section{References}

1. Fankhauser CD, Hermanns T, Lieger K (2018) Extracorporeal shock wave lithotripsy versus flexible ureterorenoscopy in the treatment of renal calculi. Clin Kidney J 11(3): 364-369.

2. Paumgartner G, Sauter GH (2005) Extracorporeal shock wave lithotripsy of gallstones $20^{\text {th }}$ anniversary of the first treatment. Eur J Gastroenterol Hepatol 17(5): 525-527.

3. Dedes V, Stergioulas A, Kipreos G (2018) Effectiveness and Safety of Shockwave Therapy in Tendinopathies. Mater Sociomed 30 (2): 131146.

4. Chung B, Wiley JP (2002) Extracorporeal shockwave therapy a review. Sports Med 32(13): 851-865.

5. Schaden W, Thiele R (2007) Shock wave therapy for acute and chronic soft tissue wounds: a feasibility study. J Surg Res 143(1): 1-12.

6. Wang CJ (2001) Treatment of nonunions of long bone fractures with shock waves. Clin Orthop Relat Dis 387: 95-101.

7. Xu ZH, Jiang Q (2009) Extracorporeal shock wave treatment in nonunions of long bone fractures. IntOrthop 33(3): 789-793.

8. Elster EA (2010) Extracorporeal shock wave therapy for nonunion of the tibia. J Orthop Trauma 24(3): 133-141.

9. Fraira R, Berta L (2011) Biological effects of extracorporeal shock waves on fibroblasts: A review. Muscles Ligaments Tendons J 1(4): 138 -147.

10. Nishida T (2004) Extracorporeal cardiac shock wave therapy markedly ameliorates ischaemia induced myocardial dysfunction in pigs in vivo. Circ Am Heart Assoc 110(19): 3055-61.

11. Gutersohn A (2000) Upregulation of vascular endothelial growth factor mRNA n human umbilical vascular endothelial cells via shock waves. Eur J Heart Failure 2(S1): 42.

12. Zhang X (2014) The dose effect relationship in extracorporeal shock wave therapy: the optimal parameter for extracorporeal shock wave therapy. J Surg Res 186(1): 484-492.
13. Wang CJ (2003) Shock wave therapy induces neovascularization in the tendon bone junction. A study in rabbits J Orthop Res 21(6): 984-989.

14. Burneikaite G, Shkolnik E, Celutukiene J (2017) Cardiac shock wave therapy in the treatment of coronary artery diseas: systematic review and meta analysis. Cardiovascular Ultrasound15: 11.

15. Yang W, He Y, Gan L (2018) Cardiac shock wave therapy promotes arteriogenesis of coronary micrangium and ILK is involved in the biomechanical effects by proteomic analysis. Sci Rep pp.1814.

16. Vainer J, Habets JH, Schalla S (2016) Cardiac shockwave therapy in patients with chronic refractory angina pectoris Neth. Heart J 24(5): 343-349.

17. Shkolnik E, Burneikaite G, Celutkiene J (2018) Efficacy of cardiac shock wave therapy in patients with stable angina: The design of randomized, triple blind, sham procedure controlled study. Anat J Cardiol 19(2): 100109

18. Kikuchi Y, Ito T, Shindo K (2017) P4023 A multicenter trial of extracorporeal cardiac shock wave therapy for refractory angina pectoris Results from the highly advanced medical treatment in Japan. Eur Heart J 38: 1(1).

19. Chetter IC, Spak JL, Dolan P (1997) Quality of life analysis in patients with lower limb ischaemia: Suggestions for European standardization. Eur J Vasc Endovasc Surg 13(6): 597-604.

20. Ciccone M, Notarnicola A (2012) Shockwave therapy in patients with peripheral artery disease. Adv Ther 29(8): 698-707.

21. Tara S, Miyamota M (2014) Low energy extracorporeal shock wave therapy improves microcirculation blood flow of ischemic limbs in patients with peripheral arterial disease: Pilot study. J Nippon Med Sch 81(1): 19-27.

22. Belcaro G, Cesarone MR (2005) Effects of shock waves on microcirculation, perfusion and pain management in critical limb ischaemia Angiology 56(4): 403-407.

23. Serizawa F, Ito K (2012) Extracorporeal shock wave therapy improves the walking ability of patients with peripheral artery disease and intermittent claudication. Circ J 76(6): 1486-1493.

24. Cayton T, Harwood AE, Smith GE (2017) Extracorporeal shockwave therapy for the treatment of lower limb intermittent claudication: study protocol for a randomized controlled trial (the Shockwave 1 trial). 18: 104.

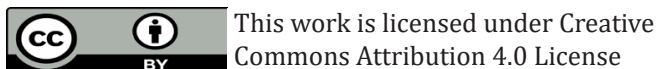

To Submit Your Article Click Here:

Submit Article

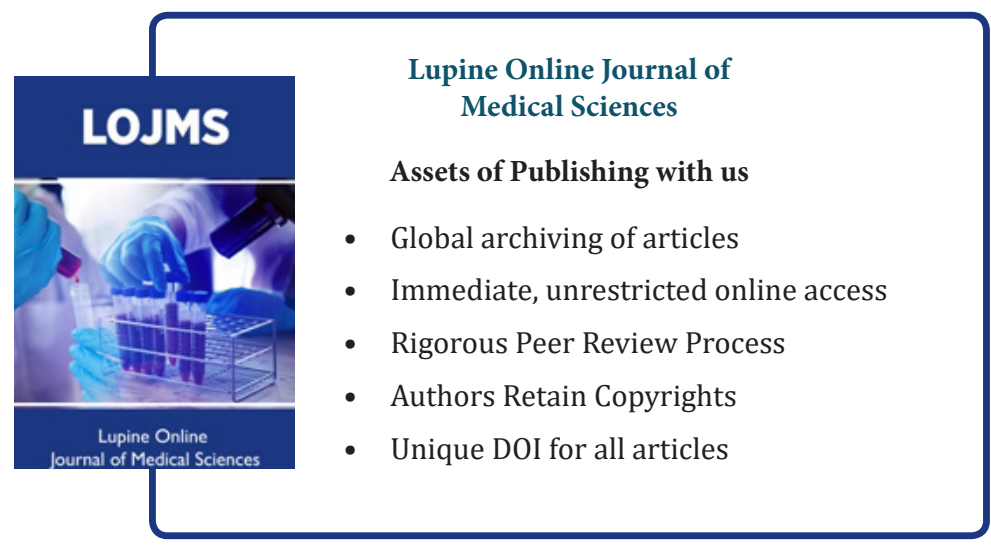

